Int. J. Dev. Biol. 55: 527-534

doi: $10.1387 / \mathrm{ijdb} .103228 \mathrm{j} 1$

\title{
Emerging paradigms and questions on pro-angiogenic bone marrow-derived myelomonocytic cells
}

\author{
JULIEN LAURENT ${ }^{1}$, CÉDRICTOUVREY ${ }^{1}$, FRANCESCA BOTTA ${ }^{1}$, \\ FRANÇOIS KUONEN ${ }^{1,2}$ and CURZIO RUEGG ${ }^{1,2,3, *}$

\begin{abstract}
${ }^{1}$ Division of Experimental Oncology (DEO), Centre Pluridiscipliniaire d'Oncologie (CePO), Centre Hospitalier Universitaire Vaudois (CHUV) and Faculty of Biology and Medicine, University of Lausanne (UNIL), Lausanne, ${ }^{2}$ National Center for Competence in Research (NCCR) Molecular Oncology, Swiss Institute of Experimental Cancer Research, Ecole Polytechnique Fédérale de Lausanne (ISREC-EPFL), Lausanne and ${ }^{3}$ Department of Medicine, Faculty of Science, University of Fribourg (UNIFR), Fribourg, Switzerland
\end{abstract}

\begin{abstract}
Cancer-related inflammation has emerged in recent years as a major event contributing to tumor angiogenesis, tumor progression and metastasis formation. Bone marrow-derived and inflammatory cells promote tumor angiogenesis by providing endothelial progenitor cells that differentiate into mature endothelial cells, and by secreting pro-angiogenic factors and remodeling the extracellular matrix to stimulate angiogenesis though paracrine mechanisms. Several bone marrow-derived myelonomocytic cells, including monocytes and macrophages, have been identified and characterized by several laboratories in recent years. While the central role of these cells in promoting tumor angiogenesis, tumor progression and metastasis is nowadays well established, many questions remain open and new ones are emerging. These include the relationship between their phenotype and function, the mechanisms of pro-angiogenic programming, their contribution to resistance to anti-angiogenic treatments and to metastasis and their potential clinical use as biomarkers of angiogenesis and anti-angiogenic therapies. Here, we will review phenotypical and functional aspects of bone marrow-derived myelonomocytic cells and discuss some of the current outstanding questions.
\end{abstract}

KEY WORDS: angiogenesis, cancer, bone marrow-derived cell, myelomonocytic cell, inflammation

\section{Introduction}

Malignant transformation and tumor progression requires the cell to initiate sustained proliferation, escape endogenous cell-cycle control and DNA-damage checkpoints, evade external inhibitory factors and senescence and resist cell death. This is achieved by a combination of genetic and epigenetic events affecting the cancer cell itself, in particular the activation of oncogenes and immortalizing pathways and the inactivation of tumor suppressor and anti-apoptotic genes. In recent years, however, it became evident that in order to give rise to a clinically relevant tumor, cancer cells require support from the tumor microenvironment (Hanahan and Weinberg, 2011). Resident stromal fibroblasts, infiltrating inflammatory cells, blood and lymphatic endothelial cells are the main stromal cell populations contributing to tumor progression (Lorusso and Rüegg, 2008). The formation of a tumor-associated vasculature, a process also referred to as tumor angiogenesis, is essential to tumor growth and progression. The initiation of tumor angiogenesis (an event also referred to as the angiogenic switch) is determined by the balance between the genetic status of the tumor itself (e.g. loss of p53, Ras activation), signals from

\footnotetext{
Abbreviations used in this paper: bFGF, basic fibroblasts growth factor; $\mathrm{BMD}$, bone marrow-derived; COX2, cyclooxygenase-2; DG, dendritic cells; eNOS, endothelial nitric oxide synthase; GM-CSF, colony-stimulating factor; HIF, hypoxia-inducible factor; iNOS, inducible nitric oxide synthase; LPS, lipopolysaccharide; MDSC, myeloid-derived suppressor cells; MMP, matrix metalloproteinase; NF- $\mathrm{\kappa B}$, nuclear factor-КB; NO, nitric oxide; NSAIDs, non-steroidal anti-inflammatory drugs; PDGF, platelet-derived growth factor; $\mathrm{PGE}_{2}$, prostaglandin $\mathrm{E}_{2}$; $\mathrm{PHD}$, proly hydoxylase; PlGF, placenta growth factor, RANKL, receptor activator for NF-אB ligand; RBCCs, recruited BMD circulating cells; ROS, reactive oxygen species; TAM, tumor-associated monocytes/macrophages; TEM, Tie-2-expressing monocytes; TGF- $\beta$, transforming growth factor beta; TLR, toll-like receptor; TNF, tumornecrosis factor; uPA, urokinase-type plasminogen. ac.tivator, VEGF, vascular endothelial growth factor; VEGFR, vascular endothelial growth factor receptor.
}

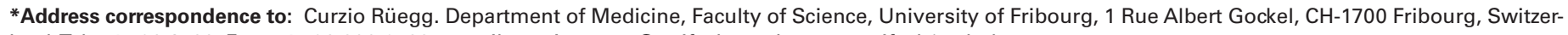
land. Tel: +41-26-8766. Fax: +41-26-300-9733. e-mail: curzio.ruegg@unifr.ch - web: www.unifr.ch/pathology
}

Final, author-corrected PDF published online: 11 July 2011

ISSN: Online 1696-3547, Print 0214-6282

(C) 2011 UBC Press

Printed in Spain 
the microenvironment (e.g. infiltrating inflammatory cells) and by the appearance of tumor hypoxia (Kerbel, 2008). Many molecular mediators and regulators of angiogenesis have been in elucidated in recent years, but our understanding of the functional relationship between angiogenic vessels and the cellular elements of the tumor microenvironment has remained limited. Cancer associated fibroblasts, immune/inflammatory cells and bone marrow-derived cells present in the tumor microenvironment concur to promote and regulate to tumor angiogenesis (Lorusso and Rüegg, 2008).

\section{Bone marrow-derived cells promoting tumor angiogenesis}

Over the past decade, efforts have been undertaken to phenotypically and functionally characterize bone marrowderived (BMD) cells recruiting to the tumor microenvironment and promoting tumor angiogenesis. Functional experiments have been performed in mice and correlative studies in human. The phenotypical and functional plasticity, two main features intrinsically associated with the nature of these cells, make these study and the interpretation of the results complex.

Angiogenesis-associated BMD cells have heterogeneous and in part overlapping phenotypes while they circulate in the blood and these phenotypes can be further modulated once the cells reach the tumor microenvironment. A further complication in the characterization of BMD/inflammatory cells is that markers used to phenotypically define these cells differ, at least in part, in human and mice. Depending of their activating stimuli, recruited BMD and inflammatory cells may have anti-tumor or tumor promoting effects. Several different BMD cell populations contributing to tumor angiogenesis have been characterized (Coffelt et al., 2010; Murdoch et al., 2008).

i) Endothelial cell progenitors, which incorporate into the vasculature and differentiate into mature endothelial cells. CD45; CD133+; CD34+, CD146.

ii) VEGFR-1 ${ }^{+}$CD $11 b^{+}$myelomonocytic cells that accumulate at tumor sites and contribute to tumor angiogenesis in a VEGFR1-dependent manner;

iii) VEGFR-1+CXCR4+CD11 b+ myeloid cells, also referred to as recruited BMD circulating cells (RBCCs), that accumulate in response to VEGF expression;

iv) $\mathrm{Gr} 1^{+} \mathrm{CD} 11 \mathrm{~b}^{+} \mathrm{BMD}$ cells, which were identified as a main source of matrix metalloproteinase (MMP) -9 ;

v) Tie-2-expressing CD11 b+ monocytes (TEM), which exert nonredundant pro-angiogenic activity through paracrine mechanisms in mice and human;

vi) Pericyte precursors, which can differentiate into mature pericytes and stabilize nascent vessels. They are described as CD11b+, Sca-1+, PDGFR9+.

\section{Myelomonocytes}
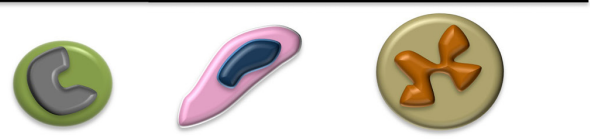

Vascular

leukocytes

MDSC

CD11 $b^{+}$

$\mathrm{CD}^{2} 1^{+}$

CD117 ${ }^{+}$

$\mathrm{CD} 117^{+}$
$\mathrm{CXCR}^{+}$

SCA1 ${ }^{+}$
CD45+

CD45+
VEGFR-1

VEGFR-2

Tie2 $^{+}$

$\begin{array}{ll}\mathrm{CD}^{+} 4^{+} & \text {VEG } \\ \mathrm{CD}^{\text {low }} & \text { VEGFR } \\ & \mathrm{Tie2}^{+}\end{array}$

CD45

Tie2 $^{+}$
VEGFR-2-

CD117-

CD133-

CD34-

CD11c $^{+}$
CD13 $^{+}$
CD16 $^{+}$
CD33 $^{+}$
CCR5 $^{+}$
CSF1R $^{+}$
CD62L $^{-}$
CD146-
CCR2

\begin{tabular}{|c|}
\hline $\begin{array}{l}\text { CD11 } \\
\text { HLA-DR } \\
\text { VE-CAD } \\
\text { ( }\end{array}$ \\
\hline
\end{tabular}

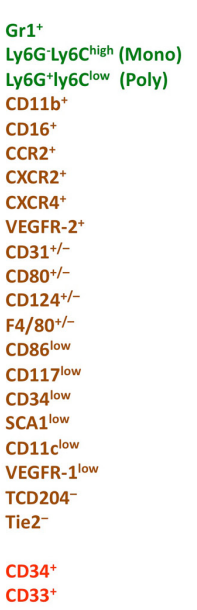

Gr1

Ly6G-Ly6C ${ }^{\text {high }}$ (Mono)

Ly6G+1

CD16

$\mathrm{CCR2}^{+}$

CXCR

VEGFR-2

$\mathrm{CD}^{+1-}$

$\mathrm{CD} 80^{+/-}$

$\mathrm{F} 4 / 80^{+/}$

CD86 low

CD117 10

SCA1 ${ }^{\text {low }}$

CD11 clow

TCD204-

$\mathrm{CD}^{\mathrm{C}} 4^{+}$
$\mathrm{CD}^{+} 3^{+}$

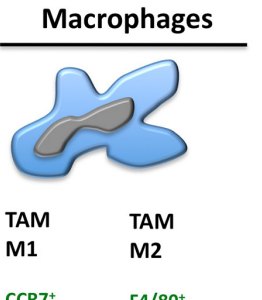

$\mathrm{CCR}^{+} \quad \mathrm{F} 4 / 80^{+}$

$\mathrm{CD}^{4^{+}} \quad \mathrm{CD} 11 \mathrm{~b}^{+}$

$\begin{array}{ll}\mathrm{CD} 16^{+} & \mathrm{CD} 14^{+} \\ \mathrm{CD} 32^{+} & \end{array}$

$\mathrm{CD} 45$
$\mathrm{CD}^{+}$

$\mathrm{CD}^{+} 0^{+} \mathrm{CD} \mathrm{CH}^{+}$

${\mathrm{CD} 86^{+}}^{+} \mathrm{CD}^{\circ} \mathrm{C6}^{+}$

$\mathrm{MHClI}^{+} \mathrm{CCR2}^{+}$

CSF1R ${ }^{+}$

$\mathrm{MHClI}^{+}$

VEGFR-1 ${ }^{+}$

VEGFR-2-

CD31-

CD34-

CD117-

CD133-

$\mathrm{CD}^{146^{-}}$

$\mathrm{CD}^{\circ}{ }^{+}$

CD163+

CXCR4 $^{+}$

Fig. 1. Phenotypical characteristics of bone marrow-derived myelomonocytic cells and macages in cancer. This figure summarizes the main bone marrow-derived myelomonocytic and et al., 2010; De Palma et al., 2005; Gabrilovich and Nagaraj, 2009; Mantovani et al., 2008; Murdoch et al., 2008; Murdoch et al., 2007; Passlick et al., 1989; Pollard, 2009) and references herein.

While tumor-mobilized myelomonocytic BMD cells have been globally considered to exert pro-angiogenic activities, one of the main challenges in the field is to correlate individual phenotypes with specific functional properties. Many excellent reviews discussing BMD cell populations and their role in angiogenesis have been published recently. Here we will specifically address phenotypicalfunctional aspects associated with myelomonocytic CD11b+BMD cells mobilized by and recruited to tumors.

\section{Phenotypic heterogeneity of monocytes and tumor associated macrophages}

Monocytes are released from bone marrow into the circulation and differentiate into macrophage at tissue sites. Macrophages present in the tumor microenvironment are referred to as tumor associated macrophages or TAM (Coffelt et al., 2010; Mantovani et al., 2008; Murdoch et al., 2008) (Fig. 1). Monocytes can be classified as resident and inflammatory subsets in mouse and human. Resident monocytes are found in both resting and inflamed tissues and express the chemokine receptor CX3CR1, the integrin LFA-1 ( $\alpha L \beta 2$ ), but lack expression of Ly6C, CCR2 and L-selectin. Resident monocytes patrol blood vessels, promote tissues remodeling and might differentiate into pro-angiogenic macrophages. One particular subset of circulating monocytes promoting tumor angiogenesis is the Tie2-expressing monocyte subset, or TEM (De Palma et al., 2005). In mouse, tumor-infiltrating TEM are CD45+CD11 $\mathrm{b}^{+}$. Human TEM have been described as CD11b+, CD11 ${ }^{+}$, CCR2$\mathrm{CCR}^{+}$and CD62L- subsets of CD45+CD14 ${ }^{\text {low }}$ CD $16^{+}$monocytes. 
A similar profile has been associated with resident monocytes. In mouse, inflammatory monocytes are selectively recruited at inflamed tissue (or lymph nodes) and express the cell surface protein Ly6C, which together with the related protein Ly6G forms the Gr1 marker, the chemokine receptor CCR2 and the adhesion molecule L-selectin (CD62). In human, two monocyte subsets can be defined based on CD14 and CD16 expression: CD14 ${ }^{\text {low } C D 16+~ m o n o c y t e s, ~}$ sharing a phenotype similar to that of resident mouse monocytes and $\mathrm{CD}_{14}{ }^{+} \mathrm{CD} 16^{-}$monocytes, resembling mouse inflammatory monocytes (Passlick et al., 1989).

Recently, a CD11 $\mathrm{c}^{+} \mathrm{HLA}-\mathrm{DR}^{+}$monocytic population termed vascular leukocytes, was reported to assemble into blood vessels in vivo expressing both endothelial and leukocytic/monocytic markers, including CD45, 'VE cadherin and Tie2 (Murdoch et al., 2008). These observations raise the question of whether distinct monocyte/ macrophage subsets found in tumors represent distinct lineages or are rather different differentiation states originated from a common progenitor cell. For example, in the mouse, tumor-infiltrating Tie2expressing monocytes and blood-circulating resident monocytes share gene expression gene signatures suggesting common developmental relationships and shared functions (Pucci et al., 2009).

TAM were among the first identified tumor-infiltrating leukocytes and are now the best characterized ones. TAM are often identified by the expression of CD11b and/or F4/80 in mouse and CD68 in human (Mantovani et al., 2008). Increased frequency of CD68+ TAM correlates with an increase in tumor vascularisation in human tumors. Monocytes/macrophages and their progenitors may also express VEGFR-1, CXCR-4 or CD14. Expression of classical mouse macrophage marker can be modulated by activation. Macrophages in response to environmental signals have been divided in M1 (classical activation) and M2 (alternative activation) subsets (Mantovani etal., 2008). M1 macrophages possess pro-proinflammatory and cytotoxic activities, while M2 macrophages are considered as immunosuppressive and pro-angiogenic macrophages. The role of TAM in tumor angiogenesis was demonstrated by many different experimental approaches and is nowadays well established. Besides promoting angiogenesis, a subset of myeloid cells can facilitate tumor growth and metastasis trough direct trophic or paracrine mechanisms (Pollard, 2009).

\section{Myeloid-derived suppressor cells (MDSC)}

A particular class of BMD myeloid cells consists of cells with immunosuppressive functions also called myeloid-derived suppressor cells (MDSC) (Gabrilovich and Nagaraj, 2009). MDSC are a phenotypically heterogeneous cell population globally defined in mice by the expression of CD11b and Gr1. This is a rather reductive definition since additional, different surface markers have been associated with immunosuppressive activity of MDSC, including CD80, CD115 (macrophage colony-stimulating factor receptor) or CD124 (IL-4 receptor alpha chain). Morphology has also been used to characterize mouse MDSC. Mononuclear MDSC are defined as monocytic MDSC and are CD11b+Ly6G-Ly6C ${ }^{\text {high }}$, whereas cells with multi-lobed or segmented nuclei are defined as granulocytic/ neutrophil-like MDSC and are CD11b+Ly6G+ly6Clow (Youn et al., 2008) in the mouse and CD11b+CD14-CD33 ${ }^{+}$HLA-DR in human (Ochoa et al., 2007).

Other types of cells, including neutrophils, eosinophils or dendritic cells may also express many of these markers and are reported to promote tumor angiogenesis (Coffelt et al., 2010; Murdoch et al., 2008)

\section{Functional characterization of myeloid bone marrow- derived cells in tumor angiogenesis}

\section{Tie2 expressing monocytes (TEM)}

The close proximity of TEM to the tumor vasculature observed by De Palma and colleagues, suggested that TEM might contribute to the regulation of tumor angiogenesis. Selective depletion of TEM by a suicide gene approach in mouse tumor models resulted in reduced tumor angiogenesis without impact on the recruitment of TAM or neutrophils (De Palma et al., 2005). This observation suggested that TEM are a sub-population of monocytes distinct from precursors of TAM. It was suggested that TEM stimulate angiogenesis by expressing the pro-angiogenic molecule bFGF (De Palma et al., 2005). Angiogenic activity of TEM may also be modulated by hypoxia and other cues, in particular angiopoietin-2. Exposure to both hypoxia and angiopoietin-2 markedly suppresses the release of the potent anti-angiogenic cytokine IL- 12 by human TEM, and the combined action of angiopoietin-2 and hypoxia inhibited the release of tumor-necrosis factor (TNF) an inflammatory cytokines with context-dependent pro- or anti-angiogenic activities (Murdoch et al., 2007) (Fig. 2).

\section{Tumor-associated macrophages (TAM)}

Whereas M1 macrophages are thought to suppress tumor growth by secreting cytotoxic agents, such as TNF, IL-12, reactive nitrogen (iNOS) and reactive oxygen species (ROS) (Sica et al., 2008), M2 macrophages are considered to promote tumor growth. M2 macrophages release a number of potent pro-angiogenic cytokines, such as VEGF-A, VEGF-C, TNF, IL-8 and bFGF and suppress the immune response through the secretion of immunosuppressive cytokines, including IL-10 and transforming growth factor (TGF9) (Sica et al., 2008). Additionally, TAM secrete a large panel of proteases, including urokinase-type plasminogen activator (UPA), members of the matrix metalloproteinase family (MMP-2, -7, -9 and -12) and elastases, which can contribute to the angiogenic switch. In vitro, TAM express additional pro-angiogenic factors like interleukin-19 and cyclooxygenase-2 (COX2) (Dirkx et al., 2006). Tumor hypoxia plays an important role in modulating TAM activity. TAM accumulate in hypoxic and peri-necrotic tumor regions. Hypoxic macrophages up-regulate hypoxia-inducible transcription factors (HIF) resulting in the increased transcription of a large panel of genes that regulate cell survival, cell metabolism and angiogenesis, including hepatocyte growth factor (HGF), VEGF, bFGF, tissue factor (TF) or MMP-12 (Dirkx et al., 2006) (Fig. 2).

\section{Myeloid-derived suppressor cells (MDSC)}

MDSC are defined by their ability to suppress immune responses, in particular T lymphocyte activation, in vitro and in vivo (Gabrilovich and Nagaraj, 2009). Several mechanisms have been proposed to explain this immunosuppressive effect, including release of cytokines and nitric oxide (NO), generation of reactive oxygen species (ROS), and increase activity of L-arginase (Kusmartsev and Gabrilovich, 2006). Cytokine-mediated immunosuppression involves MDSC-mediated increased production of type 2 cytokines by macrophages, such as IL-10 and TGF- $\beta$, and the down-regulation of the production of type 1 cytokines such as IL-12, thereby promoting 
the emergence of a M2 tumor-promoting phenotype. Furthermore, MDSC can produce TGF- $\beta$ in response to IL-13 stimulation, which further reduces the cytotoxic activity of T-cells. MDSC also express several factors with direct tumor-promoting activity, such as IL-6, platelet-derived growth factor (PDGF) or granulocyte macrophage colony-stimulating factor (GM-CSF) (Sauer et al., 2001). Arginase, inducible NO synthase (iNOS), ROS and COX-2 have been shown to play a critical role in mediating the immuno-suppressive activity of the MDSC (Gabrilovich and Nagaraj, 2009). Indeed, T-cell proliferation and activation depends on the availability of L-arginine which is the substrate for two enzymes, the inducible iNOS and arginase-I. MDSC express both enzymes at high levels (Gabrilovich and Nagaraj, 2009) resulting in a depletion of L-arginine in the microenvironment and consequently in an inhibition of T-cell function. ROS contribute to the immune tolerance of cytotoxic CD8 T-cells by altering antigen recognition (Nagaraj et al., 2007). COX-2 is a key factor promoting the activation of MDSC and in regulating the expression of arginase- 1 and iNOS. COX-2 mediates the production of prostaglandin $\mathrm{E}_{2}\left(\mathrm{PGE}_{2}\right)$, a potent positive regulator of tumor angiogenesis (Gabrilovich and Nagaraj, 2009). Thus, COX-2 coordinates the suppressive activity of MDSC and angiogenesis in the tumor microenvironment (Fig. 2).

\section{Other myeloid cell subpopulations}

While many studies have analyzed the role of TAM, MDSC and TEM in tumor angiogenesis, additional myeloid-derived cell populations may also contribute to this process, including neutrophils, eosinophils, mast cells and dendritic cells (DC) (Coffelt et al., 2010). For some of these cells, the mechanism by which they mediate or modulate tumor angiogenesis has been addressed, and in part elucidated, while for others it remains elusive. Tumor-associated neutrophils are a major source of MMP9, along with macrophages or mast cells, and appear to promote angiogenesis by releasing matrix-sequestered angiogenic factors, such as VEGF. Alternatively, they may themselves release VEGF via a degranulation process induced by inflammatory stimuli, such as TNF. Recently, it has been

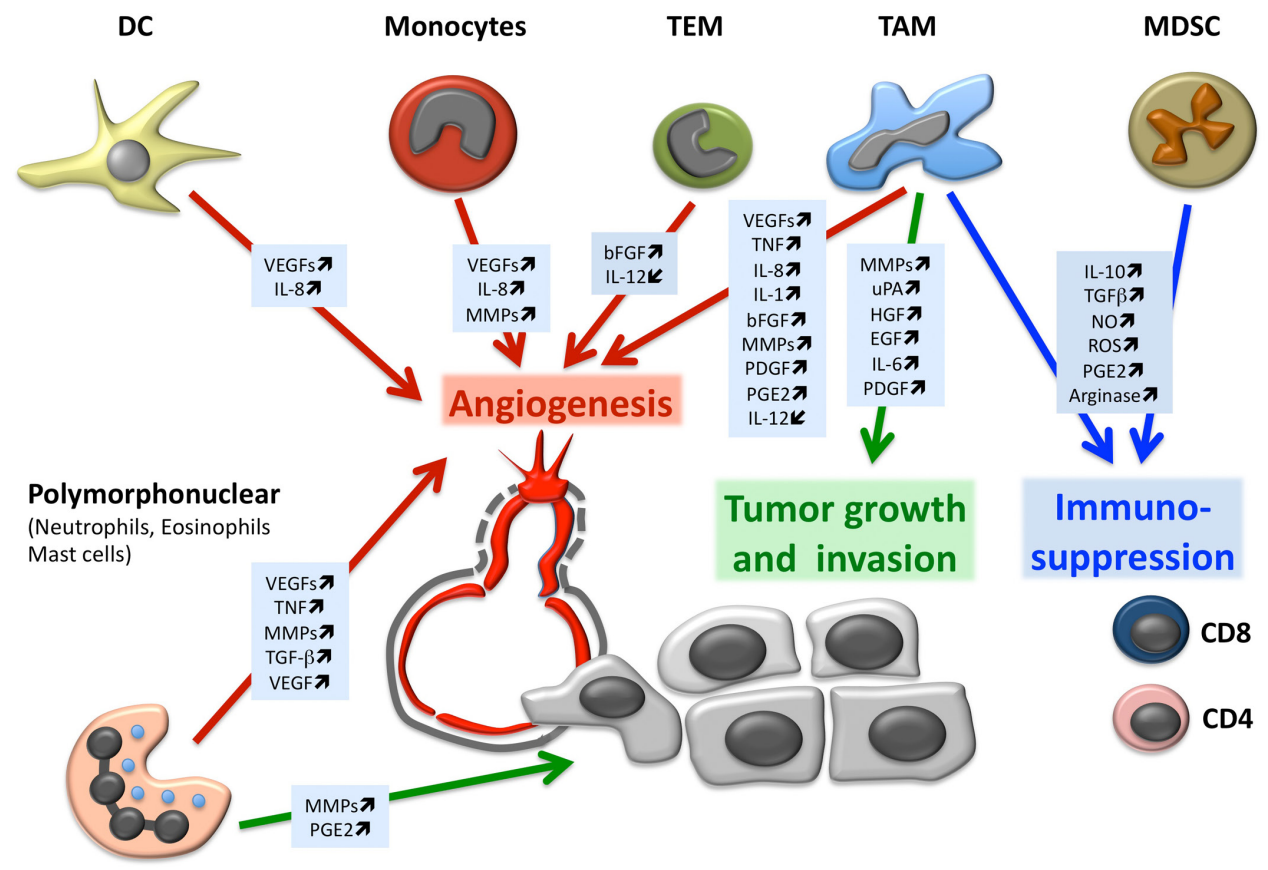

postulated that similarly to TAM, tumor-associated neutrophils can be polarized in the tumor environment into N1 or N2 phenotypes, the latter being associated to a tumor-promoting activity. TGF- $\beta$ has been identified as a factor promoting this polarization (Fridlender et al., 2009). Eosinophils and mast cells can release factors like bFGF, IL-6, IL-8, PDGF, TNF, TGF- $\beta$ and matrix-modifying proteases, in particular MMP9, with pro-angiogenci properties (Crivellato et al., 2008). In eosinophils, VEGF secretion is observed shortly after IL-15 stimulation. DC are considered to play an important role in tumor immuno-surveillance by eliciting tumor-specific $\mathrm{T}$ cells responses. Recent evidence, however, indicates that DC might also contribute to tumor angiogenesis by releasing proangiogenic factors, like VEGF and IL-8, in response to hypoxia (Ricciardi et al., 2008). Human tumor cells implanted together with immature DC have enhanced tumor vascularization and growth, while mature DC did not impact angiogenesis (Fig. 2).

These observations, together with the intrinsic plasticity of BMD and inflammatory cells in response to microenvironmental cues, and the persistence of polarizing signals in the tumor microenvironment, suggest that many, if not all BMD and inflammatory cells reaching the tumor microenvironment might eventually produce proangiogenic and pro-invasive factors promoting tumor progression.

\section{Mobilization and recruitment of bone marrow-derived myeloid cells into tumors}

Recruitment of BMD myeloid cells is a highly regulated process depending of chemo-attractant factors. CCL2, also known as monocyte chemotactic molecule-1 (MCP-1) and CCL5, also known as RANTES, are well-established chemo-attractants for myeloid cells (Balkwill, 2004). Increased levels of these chemokines lead to an increased infiltration of TAM, T-cells and eosinophils into primary tumors. The HIF-1-dependent chemokine CXCL12 (also known as stromal cell-derived factor-1, SDF-1), a ligand for the chemokine receptor CXCR4, promotes tumor angiogenesis though the mobilization and recruitment of CXCR4+VEGFR1 + hemangiocytes (Jin et al., 2006). Interleukin-8 (IL-8), VEGF, PDGF and colony-stimulating factor-1 (CSF-1) produced by various types of tumors, are also potent chemo-attractants for monocytes/macrophages. Other cytokines like IL-19 and $\beta$-defensin may also serve as recruitment factors for neutrophils, macrophages and DC (Conejo-Garcia et al., 2004). Recently, prokineticin-2, or Bv8, was identified

Fig. 2. Tumor-promoting effects of bone marrow-derived and inflammatory cells. This scheme summarizes the main bone marrow-derived and inflammatory cells present in the tumor microenvironment, which promote tumor angiogenesis, tumor proliferation, survival and invasion and suppress the specific anti-tumor immune response of CD4 and CD8 lymphocytes. Selected factors mediating these effects are indicated. Abbreviations: DC, dendritic cells; MDSC, myeloid-derived suppressor cells; TAM, tumor-associated macrophages; TEM, Tie-2 expressing monocytes. 
as a critical regulator of $C D 11 b^{+} G r 1^{+}$cell mobilization from the bone marrow, and $\mathrm{CD} 11 \mathrm{~b}+\mathrm{Gr}{ }^{+}$cell-mediated tumor angiogenesis (Shojaei et al., 2008). Anti-Bv8 antibody treatment resulted in a significant decrease of tumor growth and a reduction in $\mathrm{CD} 11 \mathrm{~b}+\mathrm{Gr} 1^{+}$ cell mobilization from the bone marrow. Placenta growth factor (PIGF), a VEGF-family member binding only to VEGFR-1, has been reported to contribute to the recruitment of myeloid cells into the tumor microenvironment (Fischer et al., 2007). Blocking PIGF results in the inhibition of monocyte/macrophage recruitment to tumor sites, reduced tumor angiogenesis and tumor growth, in particular in combination with anti-VEGF therapy (Fischer et al., 2007). The role of PIGF in tumor angiogenesis, and its value as potential therapeutic target, however, is matter of controversy (Bais et al., 2010; Van de Veire et al., 2010). Of interest, inhibition of PIGF activity results in reduced formation of lung metastases (Bais et al., 2010). MMP-9, besides being an essential mediator of the angiogenic switch through the release of matrix-bound VEGF at tumor sites, can also promote BMD cell mobilization though the release of $\mathrm{c}-\mathrm{Kit}$ ligand (or stem cell factor) form bone marrow stromal cells (Heissig et al., 2002). CSF-1 was reported to be a critical factor promoting breast cancer growth and metastasis by attracting monocytes to the turmor (Lin et al., 2001). A selective small molecule kinase inhibitor of CSF1 R(GW2580) in combination with the anti-VEGFR-2 antibody DC101 suppresses tumor growth via reduced recruitment and attenuated function of tumor-infiltrating myeloid cells (Pollard, 2009).

\section{Regulation of activation of tumor-recruited bone marrow-derived myeloid cells}

\section{Hypoxia}

One common condition present in solid tumor is hypoxia (generally $<1 \% \mathrm{pO}_{2}$ ). Tumor cells exposed to hypoxia, adapt their activities by expressing genes promoting glycolytic metabolism, angiogenesis, cell motility, invasion and metastasis. Many of these adaptive responses are regulated by hypoxia-inducible factors (HIFs) (Giaccia et al., 2004). HIFs are $\alpha / \beta$ heterodimeric transcription factors whose $\alpha$ subunit is degraded in the presence of oxygen by prolyl hydoxylases (PHDs) -mediated hydroxylation and targeting to the proteasome (Giaccia et al., 2004). HIF regulates the expression of many genes promoting angiogenesis including VEGF, PDGF-9, bFGF, eNOS, PIGF, angiopoietin-2. In addition HIF regulates the expression of chemo-attractant factors (i.e. MCP-1, CSF-1, SDF-1) to recruit myeloid cells into tumor hypoxic areas (Liao and Johnson, 2007).

\section{Inflammatory signaling pathways}

Macrophage activation depends on signals originating from the surrounding microenvironment. The NF- $\kappa \mathrm{B}$ pathway is a key mediator of macrophage activation in response to diverse environmental stimuli (i.e. stress, infections) and cancer (Karin and Greten, 2005). NF-кB activation in TAM shapes their pro-tumoral activities, including the release of TNF, IL-6 and IL-19, which sustain tumor cell growth or survival. Macrophages may change their protumoral phenotype depending of surrounding factors, tumor type, tumor stage, thereby contributing to the angiogenic switch. The role of NF-KB in the functional plasticity of TAM has been shown by several studies. Saccani et al., showed that defective of NF-кB function favored the induction of the M2 macrophage phenotype
(IL-10 $\left.0^{\text {high }} / I I-12^{\text {low }}\right)$. Nuclear overexpression of the p50 subunit resulted in the inhibition of NF-кB-dependent pro-inflammatory genes including TNF and IL-12 (Saccani et al., 2006). However, TAM from p50-deficient mice regained a pro-inflammatory (M1) phenotype with reduced tumor growth attributed to restored NF- $\mathrm{KB}$ activity (Saccani et al., 2006). Another group showed that IKK $\beta /$ $\mathrm{NF}-\kappa \mathrm{B}$ activation was required to maintain the M2 TAM phenotype in a model of ovarian cancer. Many different cell surface receptors expressed in macrophages lead to the activation of the NF- $\mathrm{KB}$ pathway, including toll-like receptor (TLR), IL-1 receptor (IL-1R) and TNF receptor 1 (TNFR-1) (Karin et al., 2006). For example, IL-19 induces the production of tumor-promoting factors, including VEGF, IL-6 and TNF in co-cultures of macrophages and B16 melanoma cells via NF- $\kappa B$. In an ovarian cancer model, macrophages from IL-1R-deficient mice failed to promote tumor growth, suggesting an important role of IL-1 signaling. Activation of TRL4 with lipopolysaccharide (LPS) in a murine cancer metastasis model induced a NF- $\kappa B$ activation and secretion of TNF by myeloid cells resulting in increased metastasis formation. Recently, Kim et al., showed that activation of TLR2 and TLR6 (receptors for Gram-positive bacteria-derived lipoteichoic acid and lipoprotein, respectively) by the proteoglycan versican up-regulated in many human tumors, induced TNF secretion by myeloid cells and increased metastasis in a Lewis lung carcinoma model (Kim et al., 2009). Further studies are necessary to investigate which factors and pathways are critically involved in the long-term maintenance of M2 phenotype of TAM and their pro-tumoral activities.

\section{Therapeutic implications}

The strong association between inflammation and cancer suggests that treatments targeting myelomonocytic inflammatory cells may have potential beneficial therapeutic effects in cancer. Several molecules and pathways have been identified and targeted in preclinical models and clinical studies (Fig. 3).

\section{cox-2}

There is solid epidemiological evidence demonstrating that the regular use of non-steroidal anti-inflammatory drugs (NSAIDs) reduces the risk of developing cancers, in particular colorectal cancer (Ulrich et al., 2006). The use of NASAIDs leads to a significant reduction in the number of colorectal polyps in patients with familial adenomatous polyposis coli (APC), thereby decreasing the risk for progression to carcinoma (Steinbach et al., 2000). These effects have been attributed to the inhibition of COX-2, the inducible pro-inflammatory cyclooxygenase isoform highly expressed in tumors. While these findings provide a proof of principle that the inhibition of tumor-associated inflammation suppresses tumor progression, the sustained inhibition of COX-2 activity in patients was associated with severe cardiovascular and thromboembolic complications and the notion of using COX-2 inhibitors for longterm treatment for cancer prevention was abandoned. Alternative therapeutic approaches targeting cancer-related inflammation and inflammatory cells need to be pursed.

\section{Tumor necrosis factor (TNF)}

There is a clear association between TNF expression and risk of cancer development and progression (Balkwill, 2009). Genetic or pharmacological inhibition of TNF suppresses the development 
of experimental tumors, including in the skin. TNF inhibitors, either soluble TFNR-2 (etanercept) or function blocking antibodies (infliximab), have entered clinical testing in multiple cancers, including ovary, breast and kidney. In these studies involving patients with advanced cancer, treatment was usually safe and well tolerated. There was evidence of biological activity of these TNF inhibitors with biomarkers correlating with anti-inflammatory activity (e.g. reduced CCL2 and IL-6 levels).

\section{Interleukin-1 (IL-1)}

$\mathrm{IL}-1$ is also a potentially attractive target for anti-cancer therapies as in some models it is clearly involved in tumor growth and progression, mostly indirectly by stimulating inflammation (Apte et al., 2006). Since IL-1 also stimulates the immune response, it could have dual, anti- and pro-tumoral effects. IL-1 is often highly expressed in solid tumors and polymorphism in the gene encoding $\mathrm{IL}-1 \mathrm{Ra}$, the natural endogenous inhibitor of IL-1, is associated with susceptibility to some cancers, including of the bladder and colon. There is today increasing interest in inhibiting IL-1 as anti-cancer therapy and the availability of a clinically-approved IL-1 antagonist, anakinra may open the way to clinical studies aimed at testing this therapeutic opportunity (Dinarello, 2010).

\section{CXCL12/CXCR4}

Inhibition of the CXCL12 (SDF-1) receptor CXCR4 with small molecular antagonists or peptides (e.g. CTCE-9908, AMD3100, RCP169), resulted in reduced tumor growth and metastasis in several experimental models, including breast, prostate and brain.

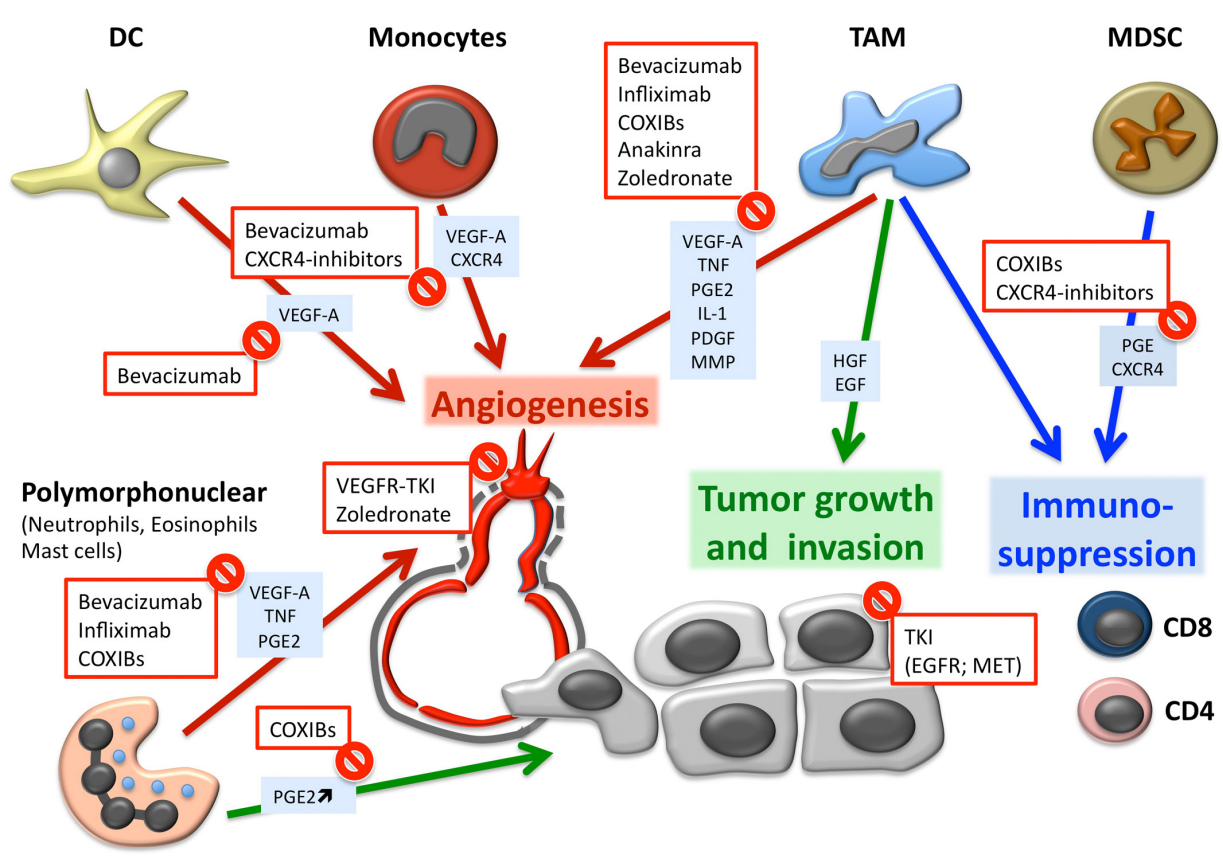

Fig. 3. Therapeutic targeting of bone marrow-derived cells. There are currently severaldrugs approved for clinical use or in development that can be used to target bone marrow-derived and inflammatory cells present in the tumor microenvironments or factors derived thereof. Some of them, such the VEGF inhibitor bevacizumab, VEGFR inhibitors (e.g. sunitinib), epidermal growth factor receptor (EGFR) inhibitors (such as the monoclonal antibody cetuximab, or the small kinase inhibitor erlotinib) are already in clinical use in oncology. Others, like the TNF inhibitor infliximab or the IL-1 inhibitor anakinra are approved for non-oncological therapies and are now being tested as anti-cancer agents. Abbreviations: COXIB, COX2 inhibitors; DC, dendritic cells; MDSC, myeloid-derived suppressor cells; TAM, tumor-associated macrophages; TEM, Tie-2 expressing monocytes; TKI, tyrosine kinase inhibitors.
CXCR4 antagonists synergize with chemotherapy to control glioma growth (Redjal et al., 2006). CXCR4 inhibition prevented accelerated tumor growth after chemotherapy by blocking the recruitment of mobilized endothelial progenitor cells and abrogated tumor regrowth after radiotherapy by preventing the influx of vasculgenic 11 b+ BMD cells into irradiated tumors (Kioi et al., 2010). Thus, reo-vascularization during tumor growth (Petit et al., 2007). Since a CXCR4 antagonist (plerixafor) is now clinically available, one may consider the design of studies in patients with

\section{Zoledonate}

Experimental evidence indicates that zoledronate and other nitrogen-containing bisphosphonates have anti-angiogenic properties. Their anti-angiogenic effects are in part mediated by direct action on endothelial cells. Treatment of endothelial cells with and capillary-like tube formation in vitro and suppressed bFGFinduced angiogenesis in vivo (Wood et al., 2002). We have shown that zoledronate acts on endothelial cells through the suppression

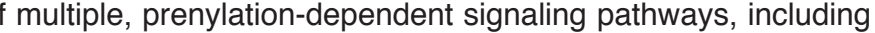
mouse model of cervical cancer, zoledronate suppressed tumor angiogenesis and tumor progression by reducing MMP-9 expression in monocytes, resulting in decreased VEGF bioavailability (Giraudo et al., 2004). Clinical studies have provided important evidence that zoledronate and other nitrogen-containing bisphosphonates may have anti-angiogenic effects in humans. Zoledronate treatment of cancer patients with bone metastases leads to a sustained reduction in serum levels of VEGF and PDGF. A transient reduction of circulating levels of bFGF and MMP-2 after zoledronate infusion was also reported. We have recently observed that in the $4 \mathrm{~T} 1$ breast tumor model, zoledronate treatments altered the phenotype of circulating BMD cells, their recruitment to tumor and decreased tumor angiogenesis (Botta, manuscript in preparation). Since zoledronate and other aminobisphosphonates are widely used in the clinics in cancer patients (i.e. for bone metastases treatment in breast and prostate cancers), it will be important to design studies to further test their ability to impinge on BMD cell mobilization and recruitment to tumors.

\section{Other drugs}

The anti-inflammatory molecule trabectedin and linomide have been described to significantly reduce tumor growth by inhibiting pro-tumoral activity TAM and/ or impaired monocytes differentiation. Several existing anticancer agents such as paclitaxel have been proposed to act, at least in part, by inhibiting TAM recruitment and function. 


\section{Outstanding questions}

It is nowadays widely accepted that BMD myelomonocytic cells are mobilized and recruited by the tumor to promote tumor angiogenesis and tumor progression. Many molecules and pathways involved in these events have been identified and investigated. Possible therapeutic targets have been further studies and clinical trials are underway to assess the feasibility of targeting BMD cells for therapeutic purposes in cancer. Nevertheless, a number of questions have remained unanswered, and novel ones are emerging. We would like to conclude by mentioning two questions of utmost clinical relevance:

Where are BMD cells programmed to acquire pro-angiogenic activity? As mentioned above, the unraveling of the correlation between BMD cell phenotype and function remain key to the understanding of the functional biology of these cells. Are there committed BMD cell populations that once induced by tumor-derived signals develop into pro-angiogenic cells by activating pre-established programs? Or are there specific tumor-derived stimuli that elicit specific differentiation program giving rise to multiple phenotypically and functionally distinct sub-populations originating from one common BMD cell precursor? Where does this education take place? It is generally assumed that cells are mobilized from the bone marrow and educated in the tumor. This seems a logical interpretation of available experimental results. Aternatively it is possible that cells are programmed in the bone marrow to express pro-angiogenic activity and to acquire a give cell surface phenotype as they are mobilized from the bone marrow. This hypothesis, however, has not been thoroughly addressed yet. Such a possibility could have important implications to tumor biology and tumor progression, since programmed cells could reach distant organs with a fully active pro-angiogenic program and thereby possibly contributing to initiate angiogenesis at pre-metastatic sites or at sites of dormant metastases. Indeed CD11 b+VEGFR-1 + myelomonocytic BMD cells have been reported to form the premetastatic niche, although their functional phenotype has not been investigated. If programming occurs before they form the pre-metastatic site, this could open the perspective of impinging on their angiogenic education before they reach the distant site.

Can BMD myelomonocytic cells be used as surrogate markers of angiogenesis? To date there are no validated biomarkers of angiogenesis that can be applied in the clinic to quantify tumor angiogenesis in individual patients and to monitor efficacy of antiangiogenic treatments. Endothelial progenitor cells have been largely explored to this end (Sessa et al., 2008). In contrast, BMD myelomonocytic cells have not been thoroughly investigated to this end. We have initiated experiments to address this question and observed specific phenotypical and functional changes in CD11 $\mathrm{b}^{+}$ myelomonocytic cells in mice (Botta etal., manuscript in preparation) and in breast cancer patients (Laurent et al., 2011) compared to tumor-free mice and healthy donors, respectively. We have also analyzed the gene expression profile of circulating myelomonocytic cells in tumor-free mice, tumor-bearing mice and tumor-bearing mice treated with anti-angiogenic drugs, and observed specific changes in gene expression (F. Botta et al., manuscript in preparation). A gene-expression-based approach to monitoring the angiogenic status of circulating myelomonocytic cells would be an attractive alternative to complex, multicolour flow cytometry-based protocols to identify rare population whose functional significance remains to be defined. Circulating myelomonocytic may emerge as candidate biomarkers angiogenesis and anti-angiogenic therapies.

\section{Conclusion}

Since the original work published by the late Jeffry Isner in 1997 and reporting progenitor endothelial cells released from the bone marrow and capable of promoting angiogenesis at distant sites (Asahara et al., 1997), we have gained a large amount of knowledge on the role of BMD cells in promoting tumor angiogenesis and cancer progression. The field has become very complex and conflicting results were sometimes reported. It is important that among this wealth of information we ask the relevant questions to unravel key issues and to identify the critical factors that may eventually allow us to translate this knowledge into therapeutic strategies in patients. Close interactions between basic and preclinical cancer researchers, clinical oncologists and industry will be necessary to reach this goal.

\section{Acknowledgments}

Work in our laboratory is supported by the Molecular Oncology Program of the National Center of Competence in Research (NCCR), a research instrument of the Swiss National Science Foundation; a Collaborative Research Grant from Oncosuisse (CCRP-OCS-01812-12-2005); a MDPhD fellowship from Oncosuisse; the MEDIC foundation, the TUMIC grant from the European Frame Program 7, and Fond'Action contre le cancer. We apologize to those colleagues whose work was not cited owing to space limitations.

\section{References}

APTE, R. N., DOTAN, S., ELKABETS, M., WHITE, M. R., REICH, E., CARMI, Y. SONG, X., DVOZKIN, T., KRELIN, Y. and VORONOV, E. (2006). The involvement of IL-1 in tumorigenesis, tumor invasiveness, metastasis and tumor-host interactions. Cancer Metastasis Rev. 25: 387-408.

ASAHARA, T., MUROHARA, T., SULLIVAN, A., SILVER, M., VAN DER ZEE, R., LI, T., WITZENBICHLER, B., SCHATTEMAN, G. and ISNER, J. M. (1997). Isolation of putative progenitor endothelial cells for angiogenesis. Science. 275: 964-967.

BAIS, C., WU, X., YAO, J., YANG, S., CRAWFORD, Y., MCCUTCHEON, K., TAN, C., KOLUMAM, G., VERNES, J. M., EASTHAM-ANDERSON, J., et al. (2010). PIGF blockade does not inhibit angiogenesis during primary tumor growth. Cell. 141: 166-177.

BALKWILL, F. (2004). Cancer and the chemokine network. Nat Rev Cancer. 4:540-550. BALKWILL, F. (2009). Tumour necrosis factor and cancer. Nat Rev Cancer. 9: 361-371.

COFFELT, S. B., LEWIS, C. E., NALDINI, L., BROWN, J. M., FERRARA, N. and DE PALMA, M. (2010). Elusive identities and overlapping phenotypes of proangiogenic myeloid cells in tumors. Am J Pathol. 176: 1564-1576.

CONEJO-GARCIA, J. R., BENENCIA, F., COURREGES, M. C., KANG, E., MOHAMED-HADLEY, A., BUCKANOVICH, R. J., HOLTZ, D. O., JENKINS, A., NA, H. ZHANG, L., WAGNER, D. S., KATSAROS, D., CAROLL, R. and COUKOS, G. (2004). Tumor-infiltrating dendritic cell precursors recruited by a beta-defensin contribute to vasculogenesis under the influence of Vegf-A. Nat Med. 10:950-958.

CRIVELLATO, E., NICO, B. and RIBATTI, D. (2008). Mast cells and tumour angiogenesis: new insight from experimental carcinogenesis. Cancer Lett. 269: 1-6.

DE PALMA, M., VENNERI, M. A., GALLI, R., SERGI SERGI, L., POLITI, L. S., SAMPAOLESI, M. and NALDINI, L. (2005). Tie2 identifies a hematopoietic lineage of proangiogenic monocytes required for tumor vessel formation and a mesenchymal population of pericyte progenitors. Cancer Cell. 8: 211-226.

DINARELLO, C. A. (2010). Why not treat human cancer with interleukin-1 blockade? Cancer Metastasis Rev. 29: 317-329.

DIRKX, A. E., OUDE EGBRINK, M. G., WAGSTAFF, J. and GRIFFIOEN, A. W. (2006). Monocyte/macrophage infiltration in tumors: modulators of angiogenesis. J Leukoc Biol. 80: 1183-1196. 
FISCHER, C., JONCKX, B., MAZZONE, M., ZACCHIGNA, S., LOGES, S., PATTARINI, L., CHORIANOPOULOS, E., LIESENBORGHS, L., KOCH, M., DE MOL, M., et al. (2007). Anti-PIGF inhibits growth of VEGF(R)-inhibitor-resistant tumors without affecting healthy vessels. Cell. 131: 463-475.

FRIDLENDER, Z. G., SUN, J., KIM, S., KAPOOR, V., CHENG, G., LING, L., WORTHEN, G. S. and ALBELDA, S. M. (2009). Polarization of tumor-associated neutrophil phenotype by TGF-beta: "N1" versus "N2" TAN. Cancer Cell. 16: 183-194.

GABRILOVICH, D. I. and NAGARAJ, S. (2009). Myeloid-derived suppressor cells as regulators of the immune system. Nat Rev Immunol. 9: 162-174.

GIACCIA, A. J., SIMON, M. C. and JOHNSON, R. (2004). The biology of hypoxia: the role of oxygen sensing in development, normal function, and disease. Genes Dev. 18: 2183-2194.

GIRAUDO, E., INOUE, M. and HANAHAN, D. (2004). An amino-bisphosphonate targets MMP-9-expressing macrophages and angiogenesis to impair cervical carcinogenesis. J Clin Invest. 114: 623-633.

HASMIM, M., BIELER, G. and RUEGG, C. (2007). Zoledronate inhibits endothelial cell adhesion, migration and survival through the suppression of multiple, prenylationdependent signaling pathways. J Thromb Haemost. 5: 166-173.

HEISSIG, B., HATTORI, K., DIAS, S., FRIEDRICH, M., FERRIS, B., HACKETT, N. R., CRYSTAL, R. G., BESMER, P., LYDEN, D., MOORE, M. A., WERB, Z. and RAFII, S. (2002). Recruitment of stem and progenitor cells from the bone marrow niche requires MMP-9 mediated release of kit-ligand. Cell. 109: 625-637.

JIN, D. K., SHIDO, K., KOPP, H. G., PETIT, I., SHMELKOV, S. V., YOUNG, L. M., HOOPER, A. T., AMANO, H., AVECILLA, S. T., HEISSIG, B. et al. (2006). Cytokinemediated deployment of SDF-1 induces revascularization through recruitment of CXCR4+ hemangiocytes. Nat Med. 12: 557-567.

KARIN, M. and GRETEN, F. R. (2005). NF-kappaB: linking inflammation and immunity to cancer development and progression. Nat Rev Immunol. 5: 749-759.

KARIN, M., LAWRENCE, T. and NIZET, V. (2006). Innate immunity gone awry: linking microbial infections to chronic inflammation and cancer. Cell. 124: 823-835.

KERBEL, R. S. (2008). Tumor angiogenesis. N Engl J Med. 358: 2039-2049.

KIM, S., TAKAHASHI, H., LIN, W. W., DESCARGUES, P., GRIVENNIKOV, S., KIM, Y., LUO, J. L. and KARIN, M. (2009). Carcinoma-produced factors activate myeloid cells through TLR2 to stimulate metastasis. Nature. 457: 102-106.

KIOI, M., VOGEL, H., SCHULTZ, G., HOFFMAN, R. M., HARSH, G. R. and BROWN, J. M. (2010). Inhibition of vasculogenesis, but not angiogenesis, prevents the recurrence of glioblastoma after irradiation in mice. J Clin Invest. 120: 694-705.

KUSMARTSEV, S. and GABRILOVICH, D. I. (2006). Role of immature myeloid cells in mechanisms of immune evasion in cancer. Cancer Immunol Immunother. 55: 237-245.

LAURENT J, HULL EF, TOUVREY C, KUONEN F, LAN Q, LORUSSO G, DOUCEY MA, CIARLONI L, IMAIZUMI N, ALGHISI GC et al. (2011). Proangiogenic Factor PIGF Programs CD11b+ Myelomonocytes in Breast Cancer during Differentiation of Their Hematopoietic Progenitors. Cancer Res. 71: 3781-3791.

LIAO, D. and JOHNSON, R. S. (2007). Hypoxia: a key regulator of angiogenesis in cancer. Cancer Metastasis Rev. 26: 281-290.

LIN, E. Y., NGUYEN, A. V., RUSSELL, R. G. and POLLARD, J. W. (2001). Colonystimulating factor 1 promotes progression of mammary tumors to malignancy. $J$ Exp Med. 193: 727-740.

LORUSSO, G. and RÜEGG, C. (2008). The tumor microenvironment and its contribution to tumor evolution toward metastasi. Histochem Cell Biol.

MANTOVANI, A., ALLAVENA, P., SICA, A. and BALKWILL, F. (2008). Cancer-related inflammation. Nature. 454: 436-444.

MURDOCH, C., MUTHANA, M., COFFELT, S. B. and LEWIS, C. E. (2008). The role of myeloid cells in the promotion of tumour angiogenesis. Nat Rev Cancer. 8:618-631.

MURDOCH, C., TAZZYMAN, S., WEBSTER, S. and LEWIS, C. E. (2007). Expression of Tie-2 by human monocytes and their responses to angiopoietin-2. J Immunol. 178: 7405-7411.
NAGARAJ, S., GUPTA, K., PISAREV, V., KINARSKY, L., SHERMAN, S., KANG, L., HERBER, D. L., SCHNECK, J. and GABRILOVICH, D. I. (2007). Altered recognition of antigen is a mechanism of CD8+ T cell tolerance in cancer. Nat Med. 13: 828-835

OCHOA, A. C., ZEA, A. H., HERNANDEZ, C. and RODRIGUEZ, P. C. (2007). Arginase, prostaglandins, and myeloid-derived suppressor cells in renal cell carcinoma. Clin Cancer Res. 13: 721s-726s.

PASSLICK, B., FLIEGER, D. and ZIEGLER-HEITBROCK, H. W. (1989). Identification and characterization of a novel monocyte subpopulation in human peripheral blood. Blood. 74: 2527-2534.

PETIT, I., JIN, D. and RAFII, S. (2007). The SDF-1-CXCR4 signaling pathway: a molecular hub modulating neo-angiogenesis. Trends Immunol. 28: 299-307.

POLLARD, J. W. (2009). Trophic macrophages in development and disease. Nat Rev Immunol. 9: 259-270.

PUCCI, F., VENNERI, M. A., BIZIATO, D., NONIS, A., MOI, D., SICA, A., DI SERIO, C., NALDINI, L. and DE PALMA, M. (2009). A distinguishing gene signature shared by tumor-infiltrating Tie2-expressing monocytes, blood "resident" monocytes, and embryonic macrophages suggests common functions and developmental relationships. Blood. 114: 901-914.

REDJAL, N., CHAN, J. A., SEGAL, R. A. and KUNG, A. L. (2006). CXCR4 inhibition synergizes with cytotoxic chemotherapy in gliomas. Clin CancerRes. 12:6765-6771.

RICCIARDI, A., ELIA, A. R., CAPPELLO, P., PUPPO, M., VANNI, C., FARDIN, P., EVA, A., MUNROE, D., WU, X., GIOVARELLI, M. and VARESIO, L. (2008). Transcriptome of hypoxic immature dendritic cells: modulation of chemokine/ receptor expression. Mol Cancer Res. 6: 175-185.

SACCANI, A., SCHIOPPA, T., PORTA, C., BISWAS, S. K., NEBULONI, M., VAGO, L. BOTTAZZI, B., COLOMBO, M. P., MANTOVANI, A. and SICA, A. (2006). p50 nuclear factor-kappaB overexpression in tumor-associated macrophages inhibits M1 inflammatory responses and antitumor resistance. Cancer Res. 66: 11432-11440.

SAUER, H., WARTENBERG, M. and HESCHELER, J. (2001). Reactive oxygen species as intracellular messengers during cell growth and differentiation. Cell Physiol Biochem. 11: 173-186.

SESSA, C., GUIBAL, A., DEL CONTE, G. and RUEGG, C. (2008). Biomarkers of angiogenesis for the development of antiangiogenic therapies in oncology: tools or decorations? Nat Clin Pract Oncol. 5: 378-391.

SHOJAEI, F., SINGH, M., THOMPSON, J. D. and FERRARA, N. (2008). Role of Bv8 in neutrophil-dependent angiogenesis in a transgenic model of cancer progression. Proc Natl Acad Sci USA. 105: 2640-2645.

SICA, A., LARGHI, P., MANCINO, A., RUBINO, L., PORTA, C., TOTARO, M. G., RIMOLDI, M., BISWAS, S. K., ALLAVENA, P. and MANTOVANI, A. (2008). Macrophage polarization in tumour progression. Semin Cancer Biol. 18: 349-355.

STEINBACH, G., LYNCH, P. M., PHILLIPS, R. K., WALLACE, M. H., HAWK, E., GORDON, G. B., WAKABAYASHI, N., SAUNDERS, B., SHEN, Y., FUJIMURA, T., SU, L. K. and LEVIN, B. (2000). The effect of celecoxib, a cyclooxygenase-2 inhibitor, in familial adenomatous polyposis. N Engl J Med. 342: 1946-1952.

ULRICH, C. M., BIGLER, J. and POTTER, J. D. (2006). Non-steroidal anti-inflammatory drugs for cancer prevention: promise, perils and pharmacogenetics. Nat Rev Cancer. 6: 130-140.

VAN DE VEIRE, S., STALMANS, I., HEINDRYCKX, F., OURA, H., TIJERAS-RABALLAND, A., SCHMIDT, T., LOGES, S., ALBRECHT, I., JONCKX, B., et al. (2010). Further pharmacological and genetic evidence for the efficacy of PIGF inhibition in cancer and eye disease. Cell. 141: 178-190.

WOOD, J., BONJEAN, K., RUETZ, S., BELLAHCENE, A., DEVY, L., FOIDART, J. M., CASTRONOVO, V. and GREEN, J. R. (2002). Novel antiangiogenic effects of the bisphosphonate compound zoledronic acid. JPharmacol Exp Ther. 302: 1055-1061.

YOUN, J. I., NAGARAJ, S., COLLAZO, M. and GABRILOVICH, D. I. (2008). Subsets of myeloid-derived suppressor cells in tumor-bearing mice. J Immunol. 181: 5791-5802. 
Further Related Reading, published previously in the Int. J. Dev. Biol.

The seminal work of Werner Risau in the study of the development of the vascular system Domenico Ribatti

Int. J. Dev. Biol. (2010) 54: 567-572

Vascular development: from precursor cells to branched arterial and venous networks Anne Eichmann, Li Yuan, Delphine Moyon, Ferdinand leNoble, Luc Pardanaud and Christiane Bréant Int. J. Dev. Biol. (2005) 49: 259-267

Parallels in invasion and angiogenesis provide pivotal points for therapeutic intervention Suzanne A. Eccles

Int. J. Dev. Biol. (2004) 48: 583-598

The "chemoinvasion assay": a tool to study tumor and endothelial cell invasion of basement membranes Adriana Albini, Roberto Benelli, Douglas M. Noonan and Claudio Brigati Int. J. Dev. Biol. (2004) 48: 563-571

The chemokine network in cancer - much more than directing cell movement Hagen Kulbe, Neil R. Levinson, Fran Balkwill and Julia L. Wilson Int. J. Dev. Biol. (2004) 48: 489-49

5 yr ISI Impact Factor $(2009)=3.253$

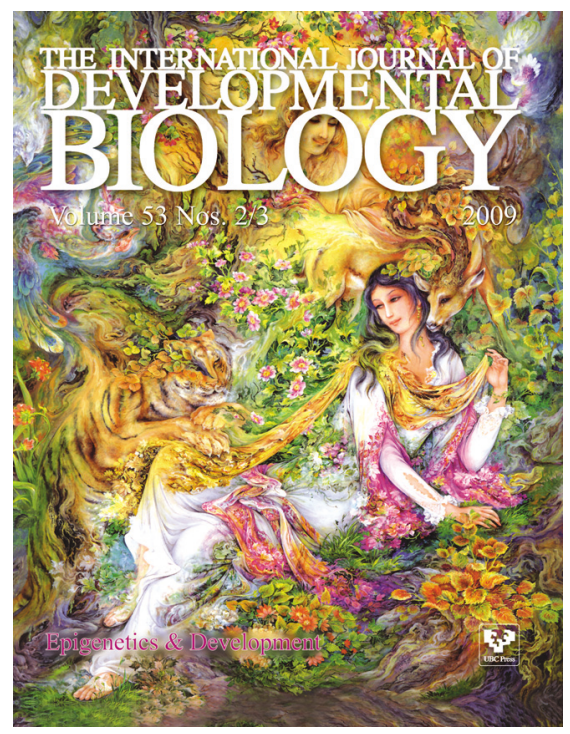

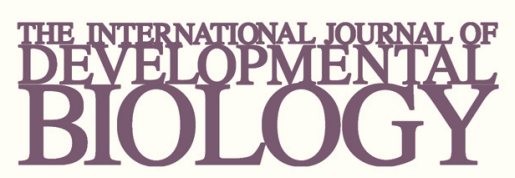

Volume 54 Nos. 6/7
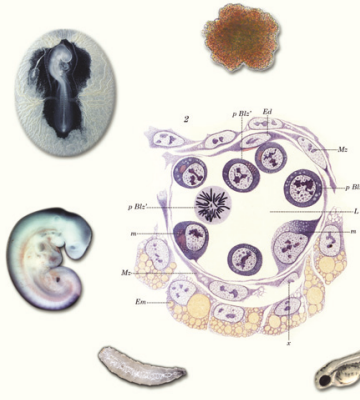

Developmental Hematopoiesis

Special Issue
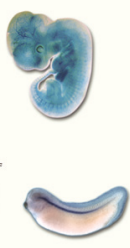

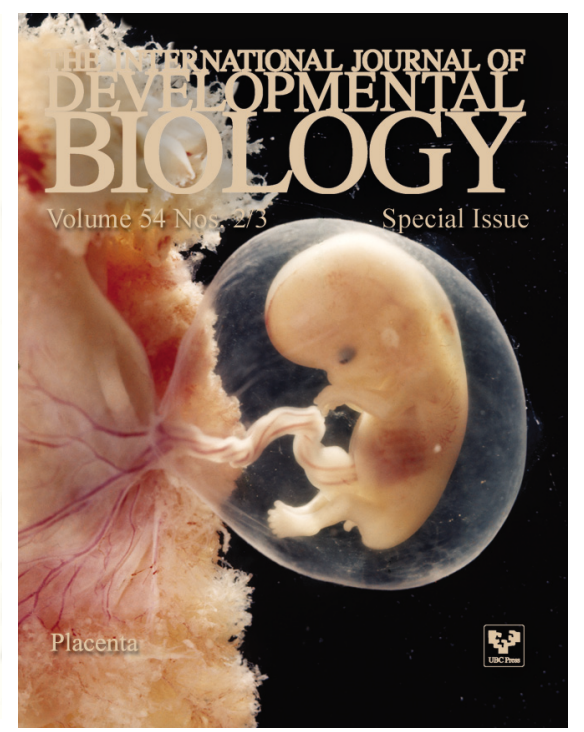

